$$
2 \omega=\frac{p}{R \rho V} \frac{\partial S}{\partial n},
$$

where $S$ is the specific entropy and $R$ is the gas constant. It can a'so be expressed simply in terms of the gradient of the total pressure $p t$ as follows:

$$
2 \omega=-\frac{p}{p_{t} \rho V} \frac{\partial p t}{\partial n} .
$$

7. The essence of the method of characteristics is the following. Suppose the flow is to be determined in the channel of Fig. 4 and the flow is known up to the line $l$. Select points $A$ and $B$ on line $l$. At these points the velocity, the direction of flow, etc., is known. Thus characteristics $C_{a}$ and $C_{b}$ can be drawn. Equation (17) used along the two different characteristics gives two equations for the velocity and its direction at point $C$. Thus properties of the flow at $C$ can be determined by solving these simultaneous equations. By repeating this process the complete flow field can be computed or constructed.

\title{
CONTINUOUS HEATING OF A HOLLOW CYLINDER*
}

\section{By G. COMENETZ** (Geophysical Laboratory, Carnegie Institution of Washington)}

1. Introduction. Formulas are given here for the temperatures within the wall of an infinitely long hollow cylinder which is supplied with heat through the inner surface. The thermal coefficients are assumed constant, the initial temperature of the wall is zero, and the outer surface is either maintained at zero or thermally insulated. Heat is transmitted at any one time uniformly all over the inner surface, but the rate of heat input is permitted to vary with the time, linearly, or at most quadratically. This, of course, is far from a general mode of variation. However, in an application in which a linear or quadratic rate is at least a permissible approximation, the formulas will be of value. They have been useful in connection with the heating of a gun firing steadily, in which, as the barrel grows hot, the heat input through the bore surface decreases nearly enough linearly with the time. Other possible applications are to the heating of a tubular furnace, a chimney, or the insulation on a wire carrying an electric current. It may not be out of place if the writer remarks that in his experience with physics, the occasions on which a first approximation is as much as is required are at least as numerous as those where only an exact theory will do.

The results follow quite directly from formulas given by Carslaw and Jaeger. ${ }^{1}$ Certain slowly convergent infinite series arise in the derivations, however, and the main effort below is directed toward summing these series in finite terms. The re-

* Received April 11, 1947. The information described in this article was obtained under contract OEMsr-51 with the Office of Scientific Research and Development, under the supervision of Division One of the National Defense Research Committee.

** Now at Westinghouse Research Laboratories, East Pittsburgh, Pa.

${ }^{1}$ H. S. Carslaw and J. G. Jaeger, Some two-dimensional problems in conduction of heat with circular symmetry, Proceedings of the London Mathematical Society (2) 46, 361-388 (1939) (referred to below as CJ). 
sults can be looked on as summation formulas for certain infinite series of orthogonal Bessel functions, and may have separate interest under that aspect. A generalization of one of Carslaw and Jaeger's formulas appears in Sec. 3.

In Sec. 5 we consider briefly the case of a heat input which is not continuous, but consists of a succession of discrete pulses occurring at equal intervals of time. Here again the gun is an example, for heat from each round is transferred to the barrel almost immediately as the round is fired, in a time that is small compared to the time between successive rounds, even at the most rapid machine-gun rate of fire. A definition, resting on a conjecture, is laid down of "equivalent steady input temperature," and an approximate relation is stated between this and the actual temperature produced by the discrete pulses. The considerations of this section relate directly only to the case of a semi-infinite solid. They can, however, be used to make initial approximations in a problem of heat transfer by uniform pulses across a surface of any shape, cylindrical or other, provided the least radius of curvature of the surface is large compared to the distance the temperature peak can travel through the given material in the time between pulses.

The writer wishes to thank Mr. Chester Snow, of the National Bureau of Standards, for some valuable suggestions, particularly in regard to proofs.

2. Outer surface at zero, input quadratic in time. Let the initial temperature of the hollow cylinder be zero, the outer surface be maintained at temperature zero, and heat be supplied across the inner surface, beginning at the initial time $t=0$, at a rate which is expressed by a quadratic polynomial in the time:

$$
q(t)=c_{0}+c_{1} t+c_{2} t^{2},
$$

where $c_{0}, c_{1}$ and $c_{2}$ are given constants. The problem is to find the temperature $\theta$ throughout the wall as a function of the radius $r$ and the time $t$, for $a \leqq r \leqq b$ and $t>0$.

Carslaw and Jaeger derived the following formula ${ }^{2}$ for a problem otherwise the same, but where the heat input, instead of being given by (1), consists of a single pulse of strength $B$ at the initial time, $t=0$, the inner surface being insulated for $t>0$. The temperature $\theta$ is thus given by

$$
\theta=\frac{\pi^{2} a B}{2 \rho c} \sum_{s=1}^{\infty} \cdot e^{-\alpha \beta_{s}^{2} t} A_{s}(r),
$$

where $\rho c$ denotes the specific heat (per unit volume), $\alpha$ the diffusivity,

$$
A_{s}(r)=\frac{\beta_{s}^{2} J_{1}^{2}\left(a \beta_{s}\right) C\left(a \beta_{s}, b \beta_{s}\right) C\left(r \beta_{s}, b \beta_{s}\right)}{J_{1}^{2}\left(a \beta_{s}\right)-J_{0}^{2}\left(b \beta_{s}\right)},
$$

$\beta_{1}, \beta_{2}, \cdots$ are the successive positive roots $^{3}$ of

$$
J_{0}\left(b \beta_{s}\right) Y_{1}\left(a \beta_{s}\right)-Y_{0}\left(b \beta_{s}\right) J_{1}\left(a \beta_{s}\right)=0,
$$

and

$$
C(x, y)=J_{0}(x) Y_{0}(y)-Y_{0}(x) J_{0}(y) .
$$

Let the temperature produced by the $B \mathrm{cal} / \mathrm{cm}^{2}$ entering instantaneously at $t=0$

2 CJ, p. 385, case (i) with $r^{\prime}=a$ and $h=0$. Their $Q$ is here $2 \pi a B / \rho c$.

${ }^{3}$ Tabulated in Jahnke-Emde, Table of functions, Dover Publications, New York, 1943, pp. 207-209. 
be written as $B f(r, t)$. Then the temperature produced by $q(t) \mathrm{cal} / \mathrm{cm}^{2} \mathrm{sec}$ entering continuously beginning at $t=0$ is given by

$$
\theta(t)=\int_{0}^{t} q(\tau) f(r, t-\tau) d \tau .
$$

Applying (5) with $q(t)$ expressed by (1) and $f(r, t)$ by the right-hand member of (2) divided by $B$, we find that

$$
\theta=P(r)+t Q(r)+t^{2} R(r)-c_{0} S_{1}(r, t)+c_{1} S_{2}(r, t)-2 c_{2} S_{3}(r, t),
$$

where

$$
\begin{aligned}
P(r) & =c_{0} S_{10}-c_{1} S_{20}+2 c_{2} S_{30}, \\
Q(r) & =c_{1} S_{10}-2 c_{2} S_{20}, \\
R(r) & =c_{2} S_{10}, \\
S_{j}(r, t) & =\frac{\pi^{2} a}{2 \rho c} \sum_{s=1}^{\infty} e^{-\alpha \beta_{s}^{2} t} A_{s}(r) /\left(\alpha \beta_{s}^{2}\right)^{i}, \quad j=1,2,3, \\
S_{j 0} & =S_{j}(r, 0) .
\end{aligned}
$$

Now the first three terms of the right-hand member of (6) satisfy all the conditions imposed on the temperature except the condition that the, initial temperature is zero; i.e., they satisfy the Fourier equation

$$
\frac{\partial \theta}{\partial t}=\alpha\left(\frac{\partial^{2} \theta}{\partial r^{2}}+\frac{1}{r} \frac{\partial \theta}{\partial r}\right)
$$

and the boundary conditions

$$
\theta(b, t)=0, \quad-k \frac{\partial \theta(a, t)}{\partial r}=c_{0}+c_{1} t+c_{2} t^{2},
$$

where $k$ denotes the conductivity. It is easy to verify this formally by considering large values of $t$ and taking into account that $S_{j}(r, t)$ approaches zero as $t$ increases indefinitely. It is, therefore, possible to obtain $P, Q, R$ in finite terms, as follows.

Replacing $\theta$ in (10) and (11) by $P+t Q+t^{2} R$ and equating like powers of $t$, we find that $R(r)$ is completely determined by the conditions

$$
\left(r R^{\prime}\right)^{\prime}=0, \quad R(b)=0, \quad-k R^{\prime}(a)=c_{2} .
$$

Then $Q(r)$ is determined by

$$
\left(r Q^{\prime}\right)^{\prime}=\frac{2 R r}{\alpha}, \quad Q(b)=0, \quad-k Q^{\prime}(a)=c_{1},
$$

and finally $P(r)$ by

$$
\left(r P^{\prime}\right)^{\prime}=\frac{Q r}{\alpha}, \quad P(b)=0, \quad-k P^{\prime}(a)=c_{0} .
$$

Entering the resulting expressions for $P, Q, R$ in (7), we derive closed formulas for the series $S_{j 0}$ : 


$$
\begin{aligned}
S_{10}= & \frac{a}{k} \ln \frac{b}{r}, \\
S_{20}= & \frac{-a}{4 \alpha k}\left\{\left[r^{2}+a^{2}(1+2 \mu)\right] \ln \frac{b}{r}+r^{2}-b^{2}\right\}, \\
S_{30}= & \frac{a}{128 \alpha^{2} k}\left\{2\left[r^{4}+4 a^{2} r^{2}+9 a^{4}-8 a^{2} b^{2}+8 a^{2} \mu\left(r^{2}+3 a^{2}+2 a^{2} \mu\right)\right] \ln \frac{b}{r}\right. \\
& \left.+\left[3 r^{2}-5 b^{2}+8 a^{2}(1+2 \mu)\right]\left(r^{2}-b^{2}\right)\right\},
\end{aligned}
$$

where $\mu=\ln (b / a)$.

The solution to the problem of this section is therefore given by formula (6), with symbols defined by (7), (15), (16), (17), (8) and (3), and the coefficients $\beta$ determined from (4).

Equation (15) may be written as

$$
\sum_{s=1}^{\infty} \frac{J_{1}^{2}\left(a \beta_{s}\right) C\left(a \beta_{s}, b \beta_{s}\right) C\left(r \beta_{s}, b \beta_{s}\right)}{J_{1}^{2}\left(a \beta_{s}\right)-J_{0}^{2}\left(b \beta_{s}\right)}=\frac{2}{\pi^{2}} \ln \frac{b}{r} .
$$

This is a summation formula for a particular infinite series of Bessel functions. Equations (16) and (17) are similar formulas. It may be observed that the terms of the series are orthogonal in the interval $(a, b)$ with respect to the weight function $r$. This is so because the $A_{s}(r)$ in (3) are a set of characteristic functions for a SturmLiouville problem from which (2) may be derived. ${ }^{4}$

As $x$ becomes large,

$$
\begin{aligned}
J_{0}(x) & \sim-Y_{1}(x) \sim \sqrt{\frac{2}{\pi x}} \cos \left(x-\frac{\pi}{4}\right), \\
J_{1}(x) & \sim Y_{0}(x) \sim \sqrt{\frac{2}{\pi x}} \sin \left(x-\frac{\pi}{4}\right) .
\end{aligned}
$$

From these well-known approximations, it follows for large $s$ in (3) and (4) that

$$
\begin{aligned}
\beta_{s} & \sim \pi\left(s-\frac{1}{2}\right) /(b-a) \\
A_{s}(r) & \sim 4(b-r) / \pi^{2}(a r)^{1 / 2}(b-a)^{2} .
\end{aligned}
$$

This makes it clear that the convergence of the series for $S_{j}(r, 0)$ in $(8)$ is like that of the reciprocal 2nd, 4th, and 6th powers of the integers, and therefore slow. For this reason it was desirable to sum the series in finite terms.

3. Extension of a formula of Carslaw and Jaeger. Consider a hollow cylinder initially at temperature zero. Let $B \mathrm{cal} / \mathrm{cm}^{2}$ of heat be liberated instantaneously at $t=0$ over a cylindrical surface of radius $r^{\prime}$ in the hollow cylinder. Let the heat radiate to a medium at temperature zero from the inner and outer surfaces, according to the conditions

${ }^{4}$ Cf. H. S. Carslaw, Mathematical theory of the conduction of heat, Macmillan, 1921, pp. 127-129. The pulse of heat can be treated as a limiting case of an initial temperature which has a constant value $T$ from $r=a$ to $a+\epsilon$, and zero from $a+\epsilon$ to $b$, where $\epsilon T$ remains constants as $\epsilon \rightarrow 0$. 


$$
\frac{\partial \theta}{\partial r}=h \theta \quad \text { at } \quad r=a, \quad \frac{\partial \theta}{\partial r}=-h^{\prime} \theta \text { at } \quad r=b
$$

where $h, h^{\prime}$ are constants not both zero. Then

$$
\theta=\frac{\pi^{2} r^{\prime} B}{2 \rho c} \sum_{s=1}^{\infty} e^{-\alpha \beta_{s}^{2} t} \frac{\beta_{s}^{2}\left[\beta_{s} J_{1}\left(a \beta_{s}\right)+h J_{0}\left(a \beta_{s}\right)\right]^{2} F(r) F\left(r^{\prime}\right)}{\left(h^{\prime 2}+\beta_{s}^{2}\right)\left[\beta_{8} J_{1}\left(a \beta_{s}\right)+h J_{0}\left(a \beta_{s}\right)\right]^{2}-\left(h^{2}+\beta_{s}^{2}\right)\left[\beta_{s} J_{1}\left(b \beta_{s}\right)-h^{\prime} J_{0}\left(b \beta_{s}\right)\right]^{2}},
$$

where

$$
F(x)=J_{0}\left(x \beta_{s}\right)\left\lfloor-\beta_{s} Y_{1}\left(b \beta_{s}\right)+h^{\prime} Y_{0}\left(b \beta_{s}\right)\right]+Y_{0}\left(x \beta_{s}\right)\left[\beta_{8} J_{1}\left(b \beta_{s}\right)-h^{\prime} J_{0}\left(b \beta_{s}\right)\right\rfloor
$$

and $\beta_{1}, \beta_{2}, \cdots$ are the successive positive roots of

$$
\begin{aligned}
{\left[\beta_{s} J_{1}\left(a \beta_{s}\right)+h J_{0}\left(a \beta_{s}\right)\right] } & {\left[\beta_{8} Y_{1}\left(b \beta_{s}\right)-h^{\prime} Y_{0}\left(b \beta_{s}\right)\right] } \\
& -\left[\beta_{s} J_{1}\left(b \beta_{s}\right)-h^{\prime} J_{0}\left(b \beta_{s}\right)\right]\left[\beta_{8} Y_{1}\left(a \beta_{s}\right)+h Y_{0}\left(a \beta_{s}\right)=0 .\right.
\end{aligned}
$$

If $h$ and $h^{\prime}$ are both zero the term $2 r^{\prime} B / \rho c\left(b^{2}-a^{2}\right)$ must be added to the right member of (23).

Carslaw and Jaeger ${ }^{5}$ give this formula for the case where $h=h^{\prime}$. The more general case above, which was derived by their method, would be required if the problem of the next section were to be generalized to permit radiation at the outer surface.

In particular, if the outer surface is thermally insulated, and the heat is liberated at the inner surface with that surface thereafter thermally insulated $\left(h=h^{\prime}=0, r^{\prime}=a\right)$, then

$$
\theta=B E_{0}+\frac{\pi B}{\rho c} \sum_{s=1}^{\infty} e^{-\alpha \beta_{s}^{2} t} E_{s}(r)
$$

where

$$
E_{0}=\frac{2 a}{\rho c\left(b^{2}-a^{2}\right)}, \quad E_{s}(r)=\frac{\beta_{s} J_{1}^{2}\left(b \beta_{s}\right)\left[J_{1}\left(a \beta_{s}\right) Y_{0}\left(r \beta_{s}\right)-Y_{1}\left(a \beta_{s}\right) J_{0}\left(r \beta_{s}\right)\right]}{J_{1}^{2}\left(a \beta_{s}\right)-J_{1}^{2}\left(b \beta_{s}\right)}
$$

and $\beta_{1}, \beta_{2}, \cdots$ are the successive positive roots $^{6}$ of

$$
J_{1}\left(a \beta_{s}\right) Y_{1}\left(b \beta_{s}\right)-Y_{1}\left(a \beta_{s}\right) J_{1}\left(b \beta_{s}\right)=0 .
$$

The reduction of (23) to (26) depends on the use of (28) and the identity

$$
Y_{0}(x) J_{1}(x)-Y_{1}(x) J_{0}(x)=\frac{2}{\pi x} .
$$

4. Outer surface insulated, input linear in time. Let the initial temperature of a hollow cylinder be zero, the outer surface be thermally insulated, and heat be supplied across the inner surface beginning at $t=0$ at a rate linear in the time:

$$
q(t)=c_{0}+c_{1} t .
$$

${ }^{5} \mathrm{CJ}$, p. 385, case (iii). They omit the additional term for $h=h^{\prime}=0$. It enters because the integrand in the inversion formula has a simple pole at the origin with this term as residue. Of course, the term is physically necessary.

${ }^{6}$ Tabulated in Jahnke-Emde, loc. cit., pp. 204-206. 
The problem is to find the subsequent temperature $\theta(r, t)$. The $q$ is taken as merely linear here because the resulting formulas are about as involved as those for a quadratic $q$ in the problem of Sec. 2 .

As in Sec. 2, we apply (5) with $q(t)$ given by $(30)$ and $f(r, t)$ by the right member of (26) divided by $B$. The result is.

$$
\theta=E_{0}\left(c_{0} t+\frac{1}{2} c_{1} t^{2}\right)+P(r)+t Q(r)-c_{0} S_{1}(r, t)+c_{1} S_{2}(r, t),
$$

where

$$
\begin{aligned}
P(r) & =c_{0} S_{10}-c_{1} S_{20}, \quad Q(r)=c_{1} S_{10}, \\
S_{j}(r, t) & =\frac{\pi}{\rho c} \sum_{s=1}^{\infty} e^{-\alpha \beta_{s}^{2} t} E_{s}(r) /\left(\alpha \beta_{s}^{2}\right)^{2}, \\
S_{j 0} & =S_{j}(r, 0), \quad j=1,2 .
\end{aligned}
$$

Proceeding as before, we recognize that the first three terms of the right member of (31) obey (10) with the conditions

$$
\frac{\partial \theta(b, t)}{\partial r}=0, \quad-k \frac{\partial \theta(a, t)}{\partial r}=c_{0}+c_{1} t .
$$

These lead to the conditions

$$
\begin{aligned}
& \left(r Q^{\prime}\right)^{\prime}=\frac{E_{0} c_{1} r}{\alpha}, \quad Q^{\prime}(b)=0, \quad-k Q^{\prime}(a)=c_{1}, \\
& \left(r P^{\prime}\right)^{\prime}=\frac{r}{\alpha}\left(E_{0} c_{0}+Q\right), \quad P^{\prime}(b)=0, \quad-k P^{\prime}(a)=c_{0} .
\end{aligned}
$$

However, in solving (36) for $Q(r)$ a new difficulty arises, for it develops that one of the two boundary conditions is redundant in view of the definition of $E_{0}$ in (27) and the relation $\rho c \alpha=k$. The consequence is that after full use has been made of (36) and (37), a constant of integration remains undetermined in $P(r)$. To fix its value another condition is required. We may find one as follows.

Since the outer surface is insulated, all the heat that has entered the cylinder up to any time $t$ is still there, or in symbols,

$$
\int_{0}^{t} 2 \pi a\left(c_{0}+c_{1} t\right) d t=\int_{a}^{b} 2 \pi r \rho c \theta(r, t) d r .
$$

Here again, taking $t$ large, we recognize that the relation must hold for the first three terms of the right member of (31). Substituting these terms for $\theta(r, t)$ in (38) and using the definition of $E_{0}$, we arrive at the conditions

$$
\int_{a}^{b} r Q d r=0, \quad \int_{a}^{b} r P d r=0 .
$$

The first is again redundant, but the second serves to fix the constant in $P(r)$.

With $P$ and $Q$ thus determined, the $S_{j 0}$ can be found from (32):

$$
S_{10}=\frac{E_{0}}{8 \alpha}\left(4 b^{2} \ln \frac{b}{r}+2 r^{2}+2 \mu \nu-a^{2}-3 b^{2}\right) \text {. }
$$




$$
\begin{aligned}
S_{20}= & \frac{a}{16 \alpha k\left(b^{2}-a^{2}\right)}\left\{4 b^{2} r^{2} \ln r-\nu\left(b^{2}-a^{2}-4 \mu b^{2}\right) \ln r\right. \\
& -\frac{1}{2} r^{4}-\left(b^{2}-a^{2}+4 b^{2} \ln b+2 \mu \nu\right) r^{2} \\
& \left.+\nu\left[\left(a^{2}+3 b^{2}\right) \mu+\left(b^{2}-a^{2}-4 \mu b^{2}\right) \ln b-2 \mu^{2} \nu\right]-\frac{1}{6}\left(2 a^{4}+2 a^{2} b^{2}-7 b^{4}\right)\right\},
\end{aligned}
$$

where $\nu=2 a^{2} b^{2} /\left(b^{2}-a^{2}\right)$.

The solution of the problem of this section is given by (31), with symbols defined by (32), (33), (40), (41), and (27), and the $\beta$ 's defined by (28).

Equation (40) may be written

$$
\sum_{s=1}^{\infty} E_{s}(r) / \beta_{s}^{2}=\frac{a}{4 \pi\left(b^{2}-a^{2}\right)}\left(4 b^{2} \ln \frac{b}{r}+2 r^{2}+2 \mu \nu-a^{2}-3 b^{2}\right)
$$

and similarly for (41). Here again the $E_{s}(r)$ may be shown to be orthogonal in the interval $(a, b)$ with respect to the weight function $r$. In this case a constant function is needed to complete the orthogonal set, as appears from the associated SturmLiouville problem.

As before, it can be shown from (19) that the series for $S_{10}$ and $S_{20}$ converge slowly, like the reciprocal 2 nd and 4 th powers of the integers.

5. Intermittent Heating. Consider a semi-infinite solid of constant thermal coefficients, initially at temperature zero, which receives heat through its surface in a series of equal pulses of strength $B \mathrm{cal} / \mathrm{cm}^{2}$. These are to occur at equal intervals of time, with a frequency of $n$ times per second, the first at $t=0$; and the surface is to be thermally insulated between pulses. The resulting temperatures have then a fluctuating character near the surface, but deeper in the solid the fluctuations become damped out, and the temperatures approximate more nearly to what would be produced by a continuous constant input of heat through the surface. The constant rate of what may be called the "equivalent steady input" is of course $n B \mathrm{cal} / \mathrm{cm}^{2} \mathrm{sec}$. But the best approximation to the fluctuating temperature is obtained by making the equivalent steady input begin, not at $t=0$, but at $t=-1 / 2 n$; that is, half the time of a pulse before the first pulse. The last statement is not one that the writer has been able to prove, although on the basis of numerical computation he is certain that it is at least very nearly true. Physically, the equivalent steady input temperature is what an instrument situated at a depth in the solid would report the temperature to be, if the instrument were not quick or sensitive enough to follow the individual fluctuations, but only their average in time. It is thus clear that the concept of equivalent steady input may be important in intermittent heating, and it would be of interest if the conjecture above could be proved.

The actual fluctuating temperature and the equivalent steady input temperature differ most at the surface of the semi-infinite solid, but there is a simple relation between them. It can be shown that at the instant before the $(m+1)$ st pulse, the equivalent steady input temperature (input commencing at $t=-1 / 2 n$ ) exceeds the fluctuating temperature at the surface by almost exactly

$$
1.46 B(n / \pi \rho c k)^{1 / 2}
$$

after the first three or four pulses. We omit the simple proof, which depends on standard semi-infinite solid heating formulas and Euler's summation formula. The 
value of this relation is that, together with a steady input calculation, it permits an estimate to be made of the base temperature level at the surface, upon which the effect of the $(m+1)$ st pulse is superimposed. The character of the $(m+1)$ st temperature surge can thus be calculated without the necessity of summing the separate effects of the $m$ preceding pulses.

\title{
NOTE ON RAYLEIGH'S METHOD AND THE NON-UNIFORM STRUT*
}

\author{
By H. A. LANG (Cornell University)
}

For a strut of the non-uniform flexural rigidity $B=E I(x)$ under end thrust, ${ }^{1}$ Rayleigh's method consists of estimating the lowest critical load $P_{1}$ by using an assumed deflection $y$ and computing the expression

$$
P=\frac{\int_{0}^{L} B\left(y^{\prime \prime}\right)^{2} d x}{\int_{0}^{L}\left(y^{\prime}\right)^{2} d x} .
$$

If $P_{1}^{\prime \prime}$ denotes the lowest value of $P$ obtained for an assumed deflection $y$ which satisfies the end conditions, it can be proved that $P_{1}{ }^{\prime}>P_{1}$, i.e., Rayleigh's method always leads to an overestimate.

An equivalent procedure may be based on the relation

$$
P=\frac{\int_{0}^{L}\left(y^{\prime}\right)^{2} d x}{\int_{0}^{L}\left(\frac{y^{2}}{B}\right) d x} .
$$

Equation (2) is obtained by eliminating $y^{\prime \prime}$ from (1) through use of the governing differential equation $B y^{\prime \prime}+P y=0$. The lowest critical load obtained from (2) for an assumed deflection $y$ is denoted by $P_{1}{ }^{\prime}$.

It is commonly taken for granted that (2) yields (a) an overestimate, and (b) a lower value than (1). These statements, however, are not obvious consequences of the fact that (1) gives an overestimate and no proof of them seems to have been given. A simple proof is presented here, i.e., it is shown that $P_{1}{ }^{\prime \prime}>P_{1}{ }^{\prime}>P_{1}$. The deflection $y$ is expanded in the form $y=\sum_{1}^{\infty} A_{N} Y_{N}(x)$ where $Y_{N}(x)$ is the deflection function corresponding to the $n$th mode and so satisfies $B Y_{N}{ }^{\prime \prime}+P_{N} Y_{N}=0$. Here $P_{n}$ is the $n$th critical load. The functions $Y_{n}$ are supposed ordered so that $Y_{1}$ and $P_{1}$ correspond to the first mode.

To simplify the discussion let

* Received April 10, 1947.

${ }^{1}$ For a detailed discussion see R. V. Southwell, Theory of elasticity, Oxford, 1946, pp. 446-453. 